\title{
Intelligent Acquisition System Used in Mechanical Laboratory
}

\author{
Raluca Rob ${ }^{1, a}$, Gelu Ovidiu Tirian ${ }^{1}$ and Caius Panoiu ${ }^{1}$ \\ ${ }^{1}$ Politehnica University of Timisoara, Department of Electrical Engineering and Industrial Informatics, Romania
}

\begin{abstract}
The main purpose of this paper consists in determining of the parameters which characterize the functioning of the Teves MK 60 as an ABS-ESP braking laboratory stand. This braking system model is used by the Volkswagen Golf and Bora the since 2002. The braking laboratory stand is able to simulate many operations which are able to give information concerning the ABS-ESP braking system comparing to the classical braking system. An application designed in LabVIEW comes to acquire and to process in real time the electrical signals generated by the Teves MK 60 laboratory stand.
\end{abstract}

\section{Introduction to Brake Assist System}

Brake assist represents an active vehicle safety feature composed to help drivers come to a stop more quickly during an episode of emergency braking. Studies show that when making emergency stops, about half of all drivers do not press the brake fast enough or hard enough to make full use of their vehicle braking power.

Teves MK 60 system is designed as a laboratory stand where some operations can be simulated. The functioning of ABS system can be studied on each wheel separately. As well, the ABS pump supplying and control are monitored, and the functioning of the acceleration sensor and the rotation sensor.

A LabVIEW application is designed to process in real time or can record the variation of the electrical signals generated by the laboratory stand using a data acquisition board. A very important issue is that using this application, the user can track the emergency situation and detect faults in functioning of the ABS-ESP system. The LabVIEW application consists in two separate parts: the acquisition application and the signal processing application. Using the acquisition application, 12 sets of faults simulation were implemented. Each one consists in generating of one event that represents a single acquisition. In this scope, 12 set of measurement were generated. Each measurement was saved into a ttxt file which can be loaded into the signal processing application.

\section{Description of Teves MK60 stand}

Teves MK60 laboratory stand is supplied to $230 \mathrm{~V}$ and is presented in Figure 1. The connection to testers can be accomplished using a pin couple. The faults simulation can be made by acting appropriate switches.

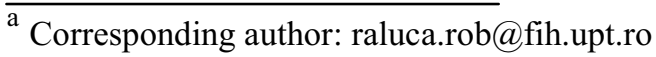

On each wheel there is a sensor that measures wheel speed. The information is transmitted to the electronic control unit. Brake lines from the master cylinder fall into two distinct channels in ABS unit. Each of these channels can be closed by an electromagnetic valve actuation. Four of the eight valves are open continuously in normal state, while the others are closed. For reasons of safety, springs systems ensure that valves remain in their normal state, if power source is disconnected.

When moderate braking occurs, braking system operates as a normal two-circuit system. Braking pressure from the master cylinder acts directly the wheel cylinder through open channels.

If the wheel tends to lock, first the valves open and close and the brake pressure accumulated to this point remains constant. If the trend lock is still too high, other valves (which were closed by this point) are open. In the ABS unit, this channel is leading to the return electric pump drive unit, which starts when these valves open. The pump sends back the brake fluid through open valves to the channel between the central cylinder and closed brake valve. The brake pressure in the wheel cylinder decreases. At the same time, the pedal is prevented from no load operation, due to the pressure in relation to the central cylinder that is constant or a bit higher. If the wheel speed begins to increase, the first valve is opened again, the second valve closes and the adjustment process repeats.

Anti-lock braking system is disabled at speeds below $6 \mathrm{~km} / \mathrm{h}$. Braking pedal pulsating is due to the low pressure fluctuation during the regulation, because of sending back of the brake fluid to the return pump. Most vehicles have two separate brake circuits with two separate brake pumps, which are powered by the same electric motor shaft.

In Figure 2 it can be seen the connector couple where the acquisition system can be attached. 


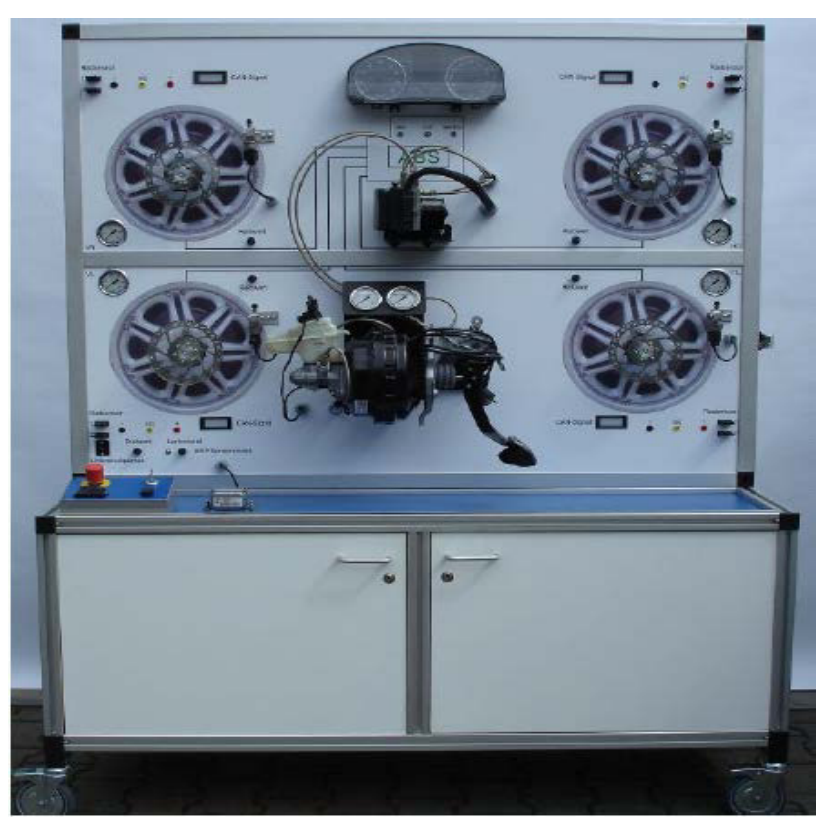

Figure 1. Teves MK60 laboratory stand.

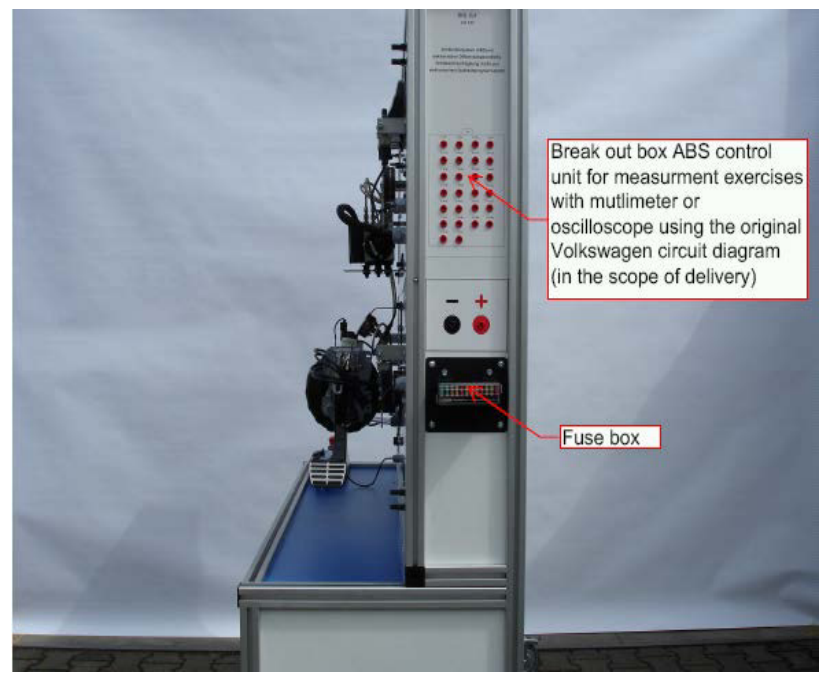

Figure 2. Connection couple for acquisition system connection.

\section{Description of laboratory acquisition device}

The data acquisition board used for acquiring the electric signals from Teves MK60 stand has the following characteristics:

-16 analogic inputs, $250 \mathrm{kS} / \mathrm{s}$ with 16 bit resolution;

-2 analogic outputs, $740 \mathrm{kS} / \mathrm{s}$ with 16 bit resolution;

-24 TTL digital in/out;

-1 digital trigger;

-Windows, Linux compatibility.

\subsection{Acquisition application}

Using the acquisition application, the voltage samples are transformed into numeric data and stored into text documents. Using DAQ Assistant.vi subroutine, 12 analogical input channels were configured in order to acquire the possible faults. The maximum sampling rate for a single channel is $20 \mathrm{kHz}$. Using a while loop, the application is able to generate and store the continuous information provided by the input signals into .txt files. The source code is presented in Figure 3.

\subsection{Signal processing application}

For each fault which is possible to occur, a measurement set was accomplished. Therefore, the signal processing application is able to load the samples from .txt files in order to process them. The faults which can be detected by the LabVIEW application consist in the followings: -ABS faults on each wheel separately -ABS pump supplying -ABS pump controlling -ABS system supplying -acceleration sensor -rotation sensor

\subsubsection{Signal processing for ABS faults}

In order to detect a fault to the ABS system on each wheel separately, each signal must be normed at input and output, as well. For filtering the signals, a nonlinear adapting filter OSLMS (Order Statistic Least Mean Square) was used. The scope of using this filter is to cancel the negaussian perturbations [1], [2].

Although the filtering, smoothing or prediction of the input signal is a linear operator depending on the input elements, the updating relations of the filter coefficients are nonlinear, so the filter that is used is considered as a nonlinear one.

It has been shown that the linear adaptive filters based on the gradient method and obtained by implementing the LMS algorithm uses an estimation of the gradient at the surface of the mean square error in order to update the filter coefficients. Such a strategy implies that the LMS algorithm is particularly vulnerable to the influence of the pulses, particularly in the case of the high amplitude pulse and short duration.

A problem that arises when using OSLMS filters is choosing the window length $L$, because the involved ordering transformations require extra calculus. [3], [4].

Generally, excepting the initialization process, one sample is changing on every step, the others remaining unchanged. For this reason, the sorting operation for updating each coefficient needs $L-1$ comparisons. For $N$ prediction coefficients, a number of $N \cdot \log _{2}(L-1)$ comparisons are required. For implementing the OSLMS filter, a number of $N \cdot L$ multiplying operations and $N \cdot(L-1)$ adding operations appear on each iteration. This facts lead to an increasing of the calculus volume proportionally to the number of the prediction coefficients $\mathrm{N}$ and also to the window length L. [5], [6], [7], [8].

It puts a question about OSLMS filter to have improved performance regarding of the noise present at the input. Such a possibility is to adjust the OSLMS filter according to a parameter that is used in order to detect the presence of a pulse which represents the fault that occurs. 


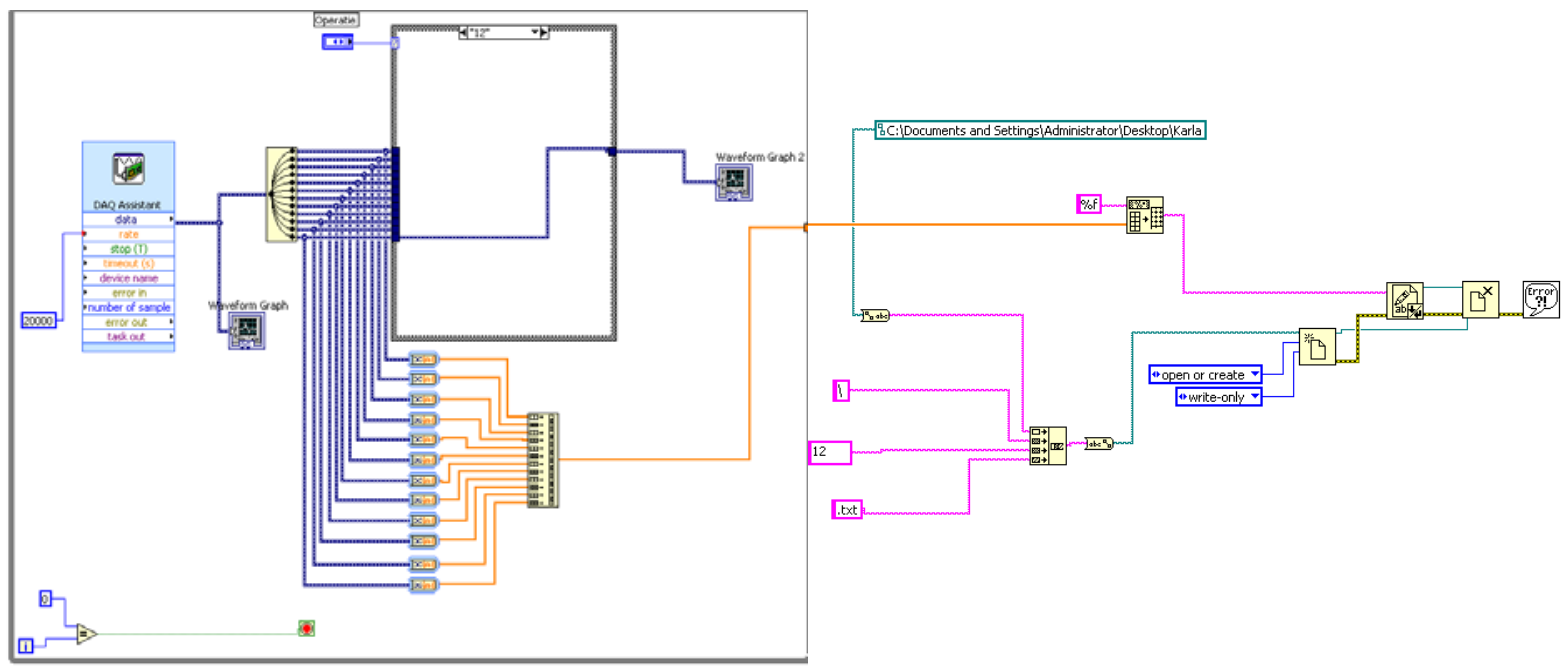

Figure 3. Source code for acquisition application.

This parameter noted with $\gamma$ is measured at each iteration using the gradient estimated in the $L$ points in the window. The parameter $\gamma$ indicates the presence or the absence of the fault inside the window and in function of the decision, the algorithm switches between the applying of the MLMS filter-Median LMS filter (if the fault occurs) or LMS filter (if there is no fault).

Considering that $x_{1}, x_{2}, \cdots, x_{L}$ are the samples from the input signal in the $L$ window length and $x_{(I)} \leq x_{(2)} \leq \cdots \leq x_{(L)}$ represents the $L$ sorted samples, the parameter $\gamma$ has the following relation:

$$
\gamma=\frac{\frac{1}{L} \cdot \sum_{i=1}^{L}\left(x_{i}-\bar{x}\right)^{4}}{\left[\frac{1}{L} \cdot \sum_{i=1}^{L}\left(x_{i}-\bar{x}\right)^{2}\right]^{2}}
$$

where $\bar{x}=\frac{1}{L} \cdot \sum_{i=1}^{L} x_{i}$ represents the average of the $L$ samples.

The method that is implemented into LabVIEW application is based on calculating the samples average from the input window at $n$ iteration, taking into consideration the samples average from the input window at $n-1$ iteration (Figure 4).

Considering that $x(n-L), x(n-L+1), \cdots, x(n-1)$ are the samples from the input window at n-1 iteration, having the average $\bar{x}_{L}(n-1), \quad$ and $x(n-L+1), x(n-L+2), \cdots, x(n)$ are the samples from $\mathrm{t}$

he input window at $n$ iteration, having the average $\bar{x}_{L}(n)$, the recurrence relation is the following :

$$
\bar{x}_{L}(n)=\bar{x}_{L}(n-1)-\frac{x(n-L)-x(n)}{L}
$$

In Figure 5 the signal variation for ABS faults with input norming, $\mathrm{L}=250$ samples is presented. Figure 6 uses the filtering algorithm for wheel 2 and Figure 7 the variation uses the output norming as well.
The LabVIEW application made possible to detect faults to ABS pump (ABS pump supplying, ABS pump command).

Pressure Release occurs when the control unit energizes the Outlet Valve while continuing to hold the Inlet Valve closed. The trapped brake fluid is released out of the calipers reducing braking pressure. At the same time the pump is switched on which draws in the released brake fluid and pumps it back into the pressure build-up circuit restoring the volume of brake fluid again in front of the Inlet valve. In Figure 8 the signal variation for ABS pump command with input norming (when the fault occurs), $\mathrm{L}=250$ samples is presented.

\subsubsection{Signal processing for faults to acceleration and rotation sensors}

For rotational speed, the rotation sensor produces a reference signal of 2.5 volts and a voltage input signal from 0.7 to 4.3 volts. This signal represents the rotational movement (yaw) of the vehicle from the neutral straight ahead position.

The sensor also integrates the transverse acceleration signal (side-ways acceleration). The signal range is $0.5 \mathrm{~V}$ increasing to $4.5 \mathrm{~V}$ as side forces (g-force) increase. This signal is combined with the rotation signal to determine when to start DSC regulation.

The signal processing is accomplished using the same OSLMS filtering algorithm. The front panel of the application which presents the variation of the signals generated by these sensors is presented in Figure 9.

\subsubsection{Signal processing for faults to CAN protocol}

CAN (Controller Area Network) is a real-time communication protocol which facilitates information exchange between car systems in order to optimize the controlling functions. If a fault occurs to CAN bus, the LabVIEW application is able to detect it and to display the signals variation. Figure 10 presents the signal variation for CAN bus and Figure 11 shows the source code for this subroutine. 


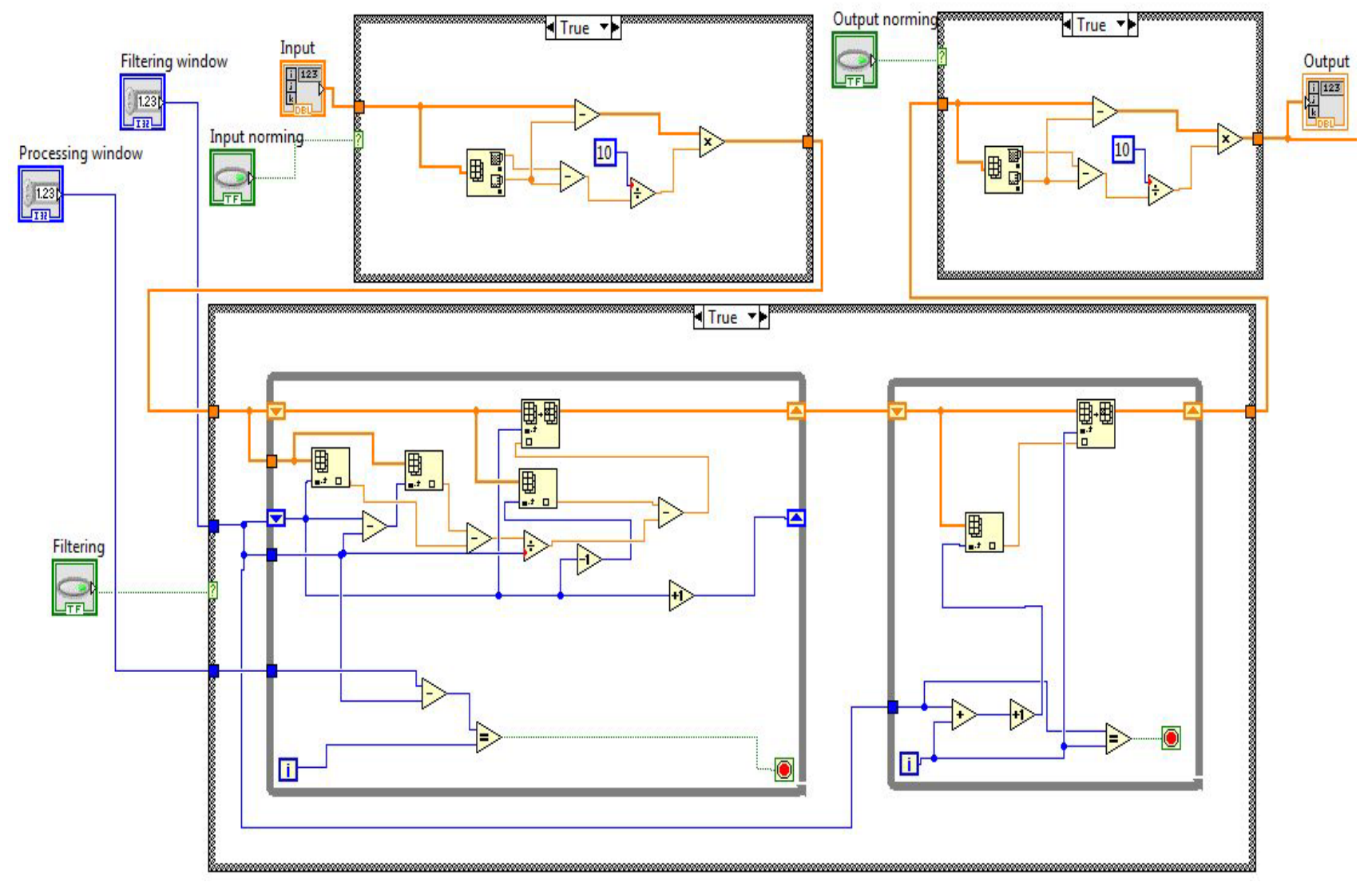

Figure 4. Source code for processing the ABS faults.

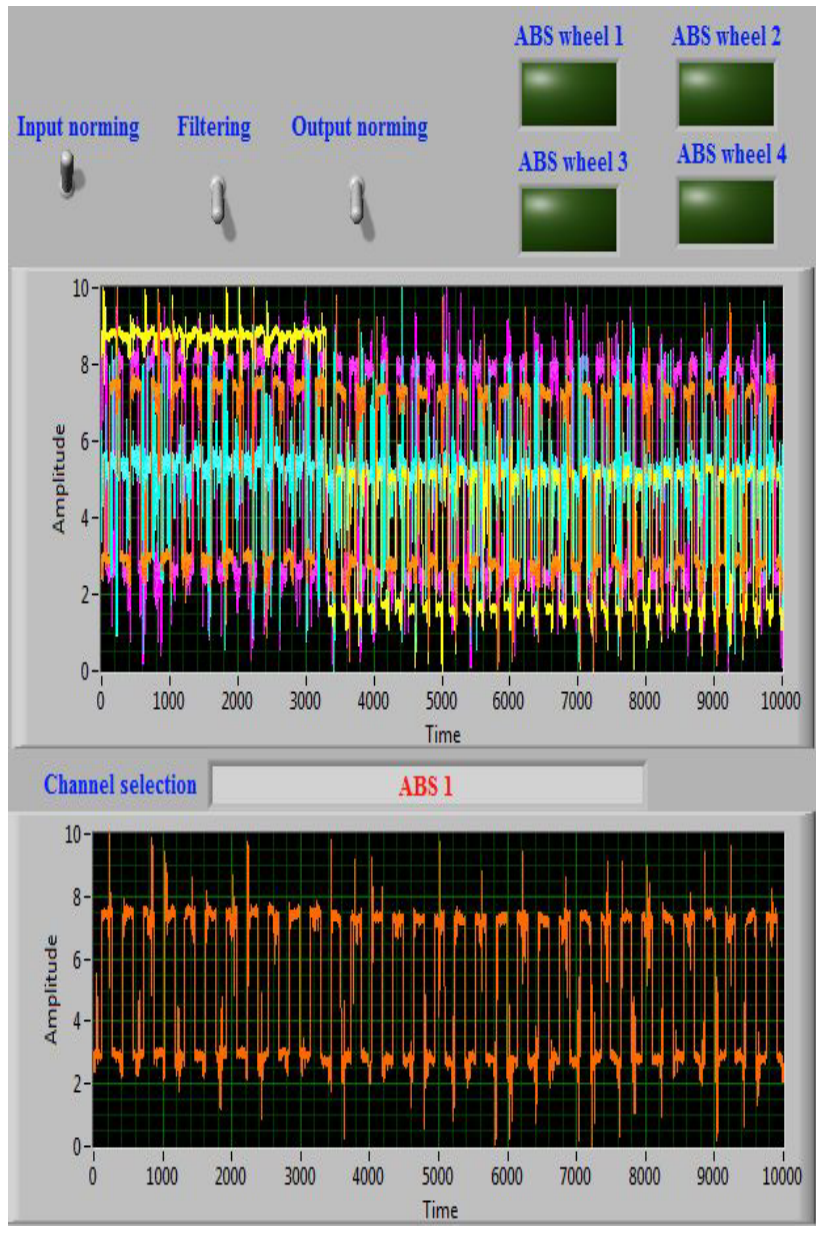

Figure 5. Signal variation for ABS faults with input norming, $\mathrm{L}=250$ samples. 


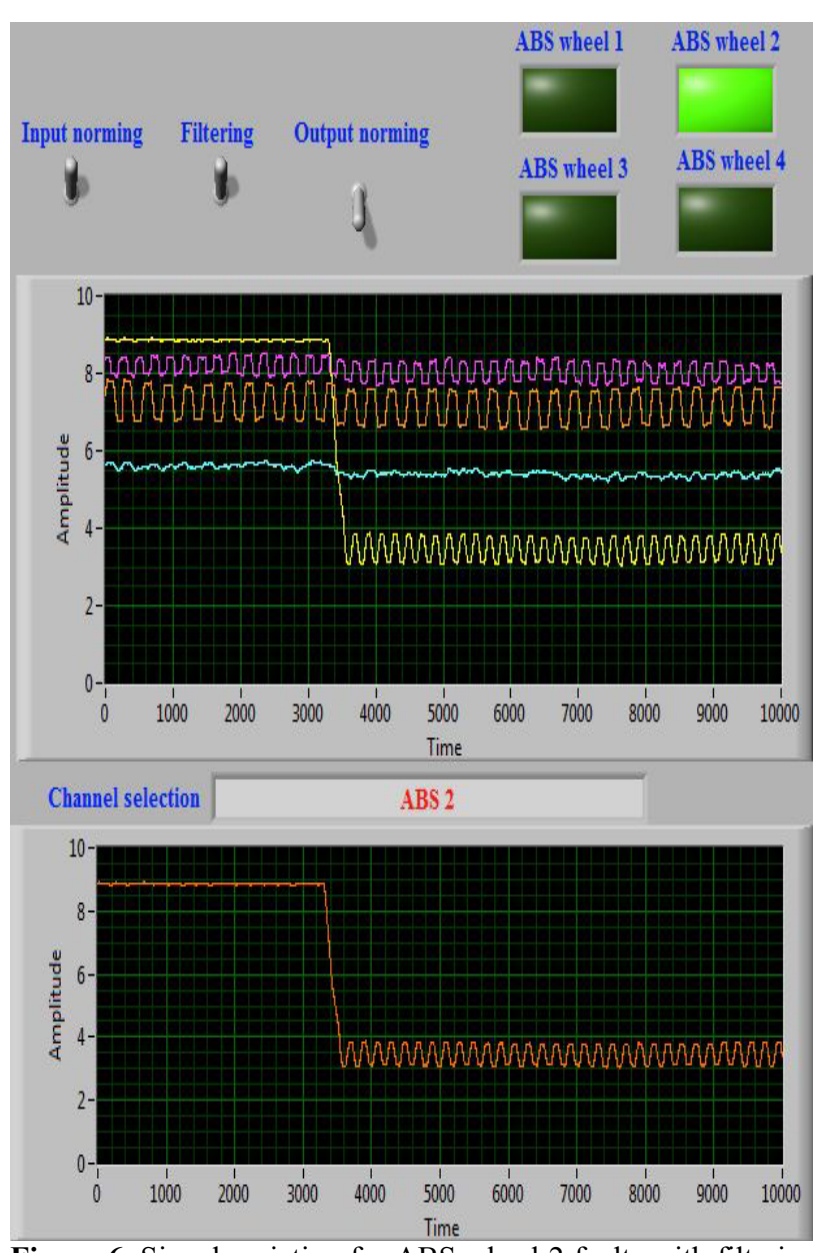

Figure 6. Signal variation for ABS wheel 2 faults with filtering (when the fault occurs), $\mathrm{L}=250$ samples.

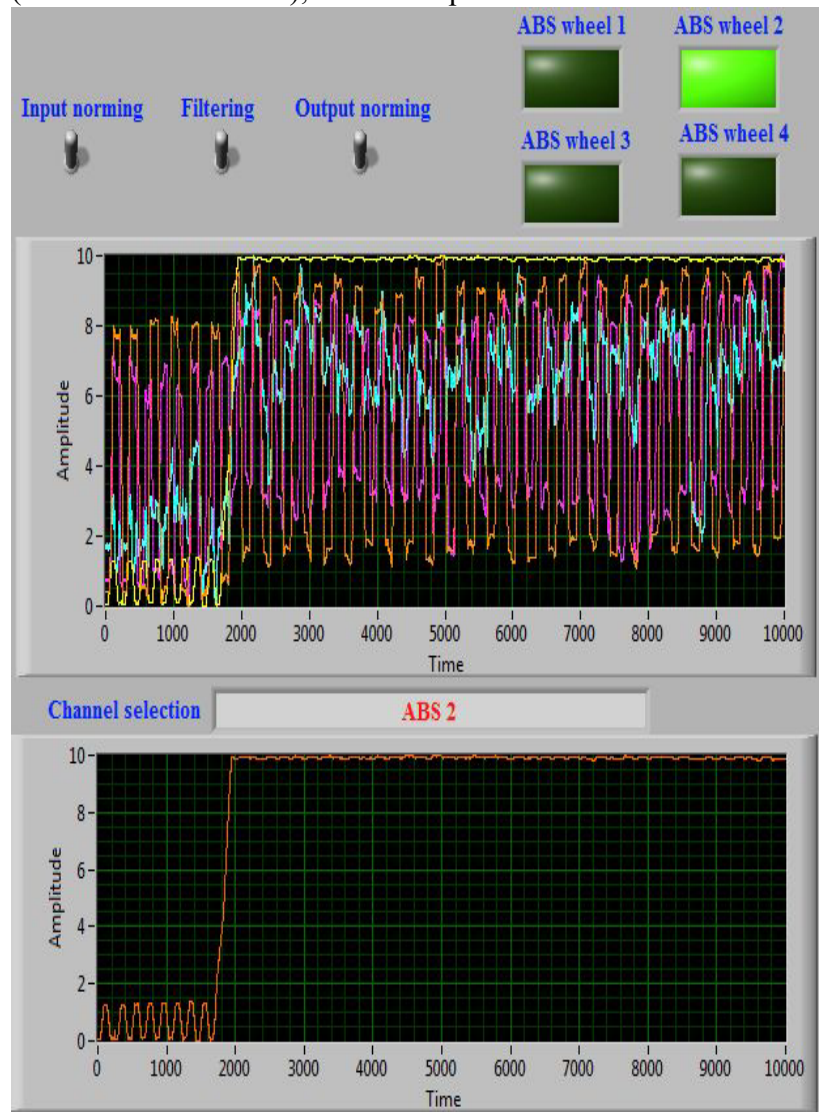

Figure 7. Signal variation for ABS wheel 2 faults with filtering and output norming (when the fault occurs), $\mathrm{L}=250$ samples.

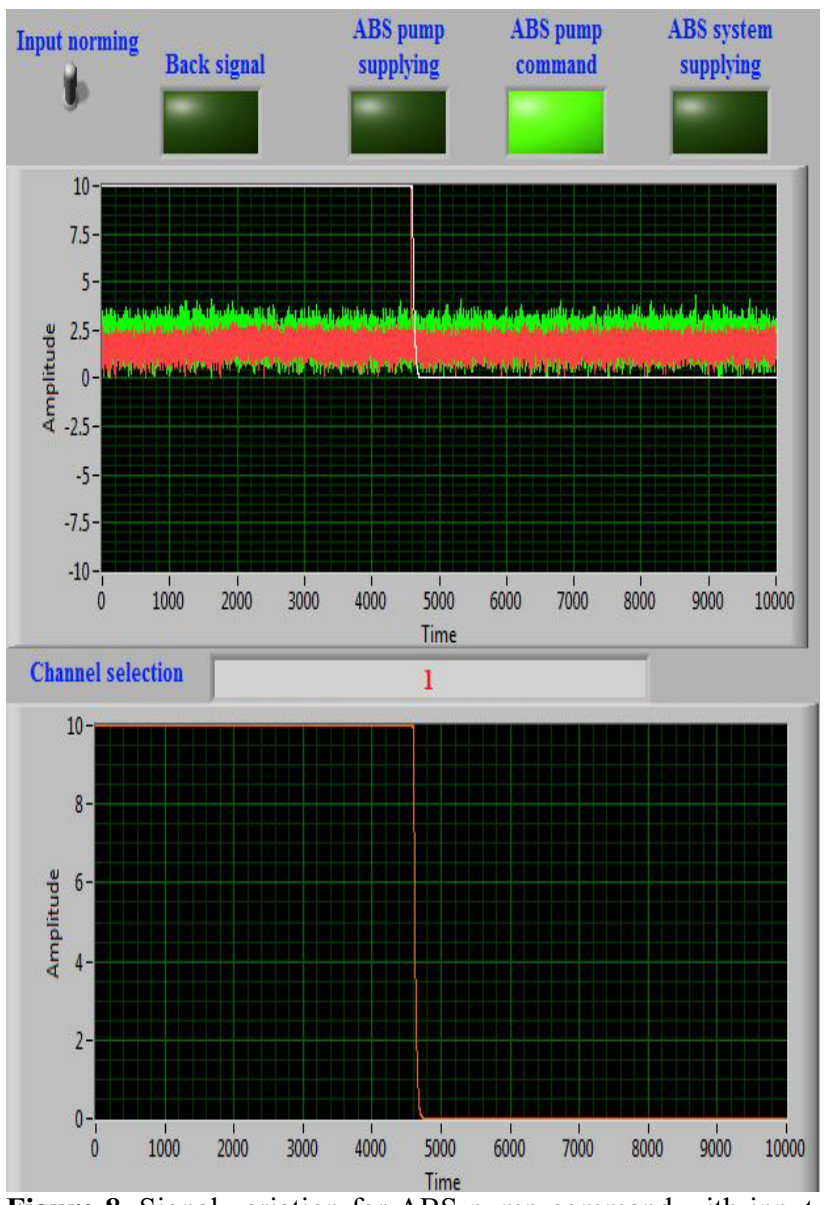

Figure 8. Signal variation for ABS pump command with input norming (when the fault occurs), $\mathrm{L}=250$ samples.
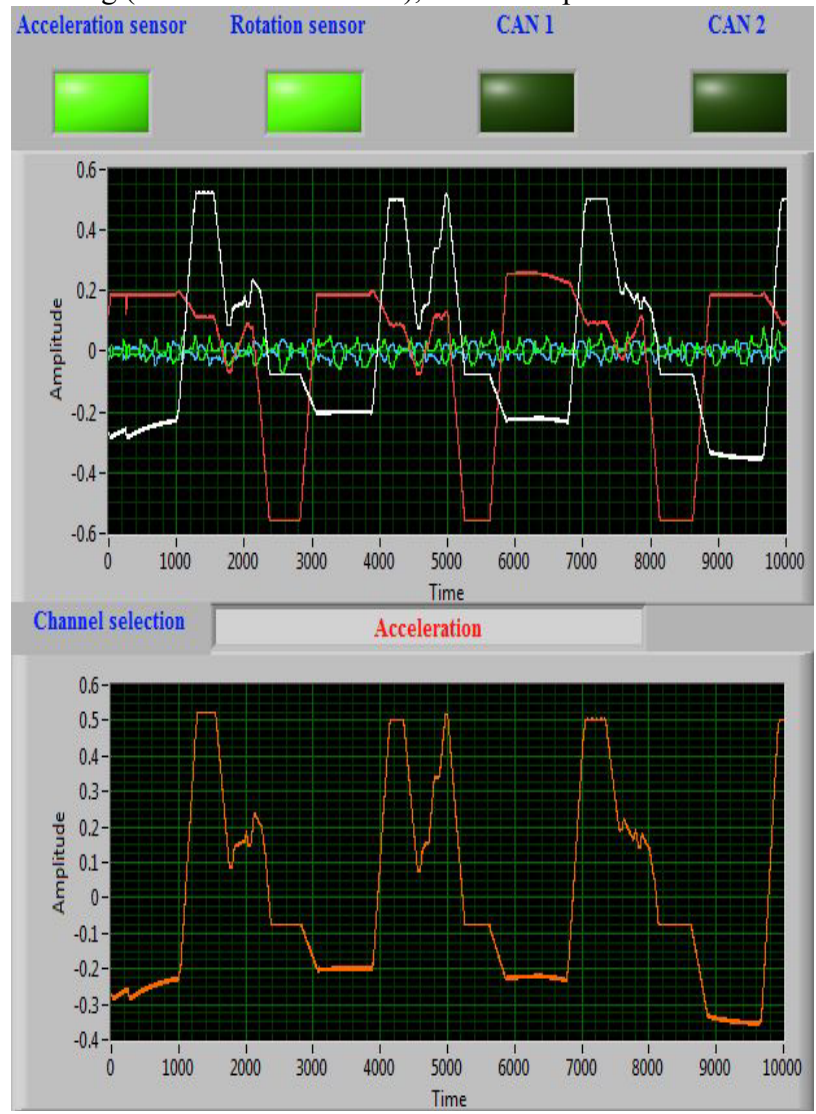

Figure 9. Signal variation for acceleration and rotation sensors (when the fault occurs), $\mathrm{L}=250$ samples. 


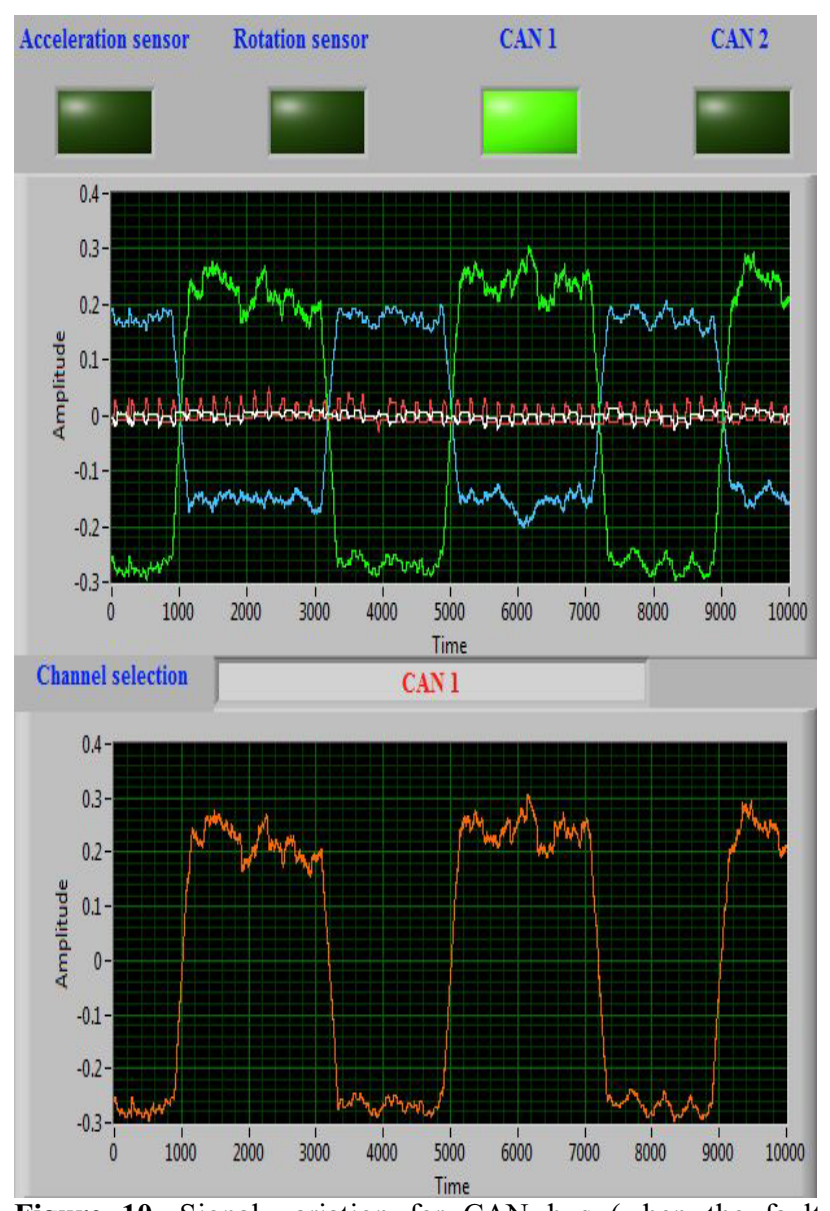

Figure 10. Signal variation for $C A N$ bus (when the fault occurs), $\mathrm{L}=250$ samples.

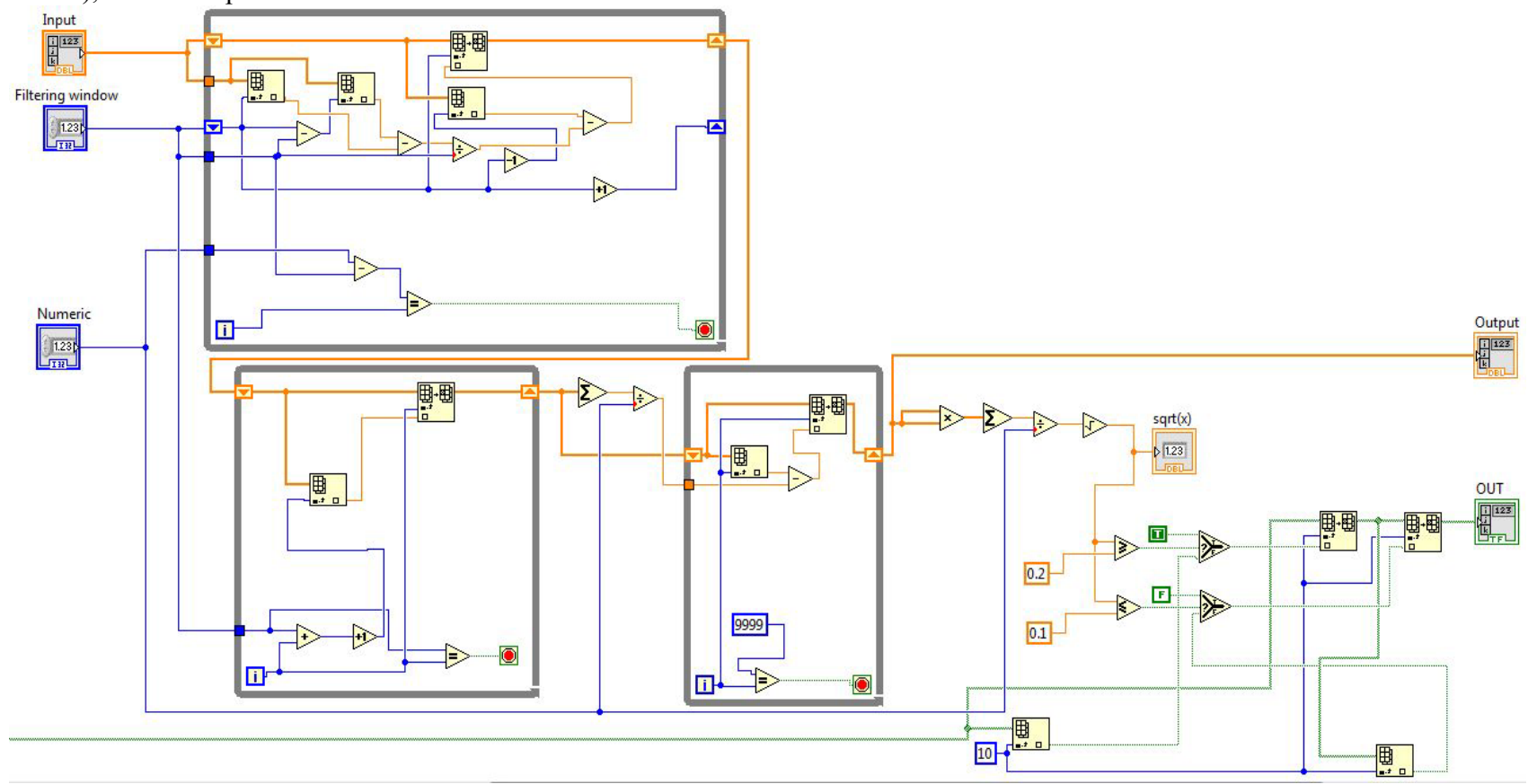

Figure 11. Source code for processing signal to CAN bus.

\section{Conclusions}

Signal processing applications are very useful in monitoring and processing data acquired from various devices. LabVIEW application described in this paper can work along with the ABS-ESP stand as an educational software.

The application provides facilities in studying the faults generated by TEVES MK60 stand. It works in real time with the stand functioning. The LabVIEW 
application is able to display the variation of the generated signal using an appropriate and facile interface with the user. The front panel of the application contains indicators which work in real time with the application.

Using the acquisition application, the information from the input signals (faults) is saved into .txt documents The algorithm of the signal processing application proposes an OSLMS filter which uses a parameter for detecting the input pulse. This method is based on computing the average of the samples from the input window at $n$ iteration, considering as known the average of the samples from the input window at $n-1$ iteration.

The signal processing application offers the possibility of comparing the results with any real testing systems in order to accomplish an educational method for mechanical students.

\section{Acknowledgement}

This work was supported by a grant of the Romanian National Authority for Scientific Research and Innovation, CNCS - UEFISCDI, project number PN-IIRU-TE-2014-4-1788.

\section{References}

1. C. Panoiu, L. Toma, M. Panoiu, R. Rob, Properties of IIR-OSLMS adaptive filters (Proceedings of the 27 th IASTED International Conference on
Modelling, Identification and Control MIC 2008, Innsbruck, Austria, pp. 460-465, 2008)

2. C. Panoiu, M. Panoiu, MALMS - A new OSLMS filter (Proceedings of the 4th IASTED International Conference Signal Processing, Pattern Recognition, and Applications, SPPRA 2007 , Innsbruck, Austria, pp.94-98, 2007)

3. http://www.bmwtech.ru/pdf/e46/ST034/14\%20P2\%2 0Traction $\% 20$ and $\% 20$ Stability $\% 20$ Control $\% 20$ Inter net.pdf

4. Sabbioni, F. Cheli, V. D'Alessandro, Analysis of ABS/ESP Control Logics Using a HIL Test Bench (SAE Technical Paper 2011-01-0032, 2011)

5. H. Witter, M. Haiden, K. Talwar, ABS/ESP ECU Testing with Sophisticated HIL Simulation Methods (Symposium on International Automotive Technology 2009)

6. Fennel H., Ding E.L., A model based failsafe system for the Continental TEVES Electronic Stability Program (ESP), SAE Technical Paper 2000-011635, 2000, doi : 10.4271/2000-01-1635.

7. Tanneli M., Friction curve peak detection by wheel deceleration measurement, 2006 IEEE Intelligent Transportation Systems Conference, pp. 1592-1597, ISSN : 2153-0009.

8. Sathyanarayana A., Boyraz P., Purohit Z., Lubag R., Driver adaptive and context aware active safety systems using CAN-bus signals, Intelligent Vehicles Symposium (IV), 2010 IEEE, pp. 1236 - 1241, ISSN: 1931-0587. 